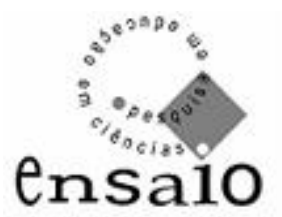

Vol. 10 no. 1 jun. 2008

\title{
Instrumentos de avaliação do ambiente de aprendizagem da sala de aula: uma revisão da literatura ${ }^{1}$
}

\author{
Instruments for the evaluation of classroom learning environment: a literature \\ review
}

\author{
Daniel Abud Seabra Matos² \\ Sérgio Dias Cirino ${ }^{3}$ \\ Walter Lana Leite4
}

\section{Resumo}

Nos últimos trinta anos, pesquisas internacionais têm se dirigido para o desenvolvimento e uso de instrumentos para avaliar o ambiente de aprendizagem da sala de aula a partir da perspectiva do aluno. Segundo Dorman (2002), a maioria das pesquisas tem sido conduzida nos Estados Unidos e na Austrália. Para Fraser (2002), as modalidades de pesquisa mais comuns são: associações entre os resultados dos alunos e o ambiente; avaliação de inovações educacionais; diferenças entre as percepções dos alunos e do professor de uma mesma sala de aula; determinantes do ambiente da sala de aula; uso de métodos de pesquisa qualitativos; e estudos transculturais. Essa revisão da literatura demonstra a riqueza de um campo de pesquisa que continua em evolução.

Palavras-chave: Percepção dos alunos, Ambiente de aprendizagem da sala de aula, Avaliação.

\footnotetext{
Abstract

In the last thirty years, international research on classroom learning environment has moved towards the development and use of instruments based on the students' perspective. According to Dorman (2002), most of this research has been conducted in the United States and Australia. Fraser (2002) indicates that research about classroom learning environments most commonly focus on: associations between student outcomes and the learning environment, evaluation of educational innovations, differences between teachers' and students' perceptions of the same classroom, determinants of classroom learning environment, utilization of qualitative methods, and cross-cultural studies. The literature review presented here demonstrates the richness of the research that has been accomplished about classroom learning environment, and indicates that this area of research is continuously evolving.

Key words: Student perceptions, Classroom learning environment, Assessment.

${ }^{1}$ Apoio: Fundação de Amparo à Pesquisa do Estado de Minas Gerais (FAPEMIG)

2 Doutorando em Educação, UFMG. Membro do LAPED, Laboratório de Psicologia e Educação da FaE/UFMG. Professor do Centro Universitário UNA.

${ }^{3}$ Professor do Programa de Pós-graduação da Faculdade de Educação da UFMG. Coordenador do LAPED, Laboratório de Psicologia e Educação da FaE/UFMG.

4. Professor do Programa de Metodologia de Pesquisa e Avaliação do Department of Educational Psychology, University of Florida, USA.
} 


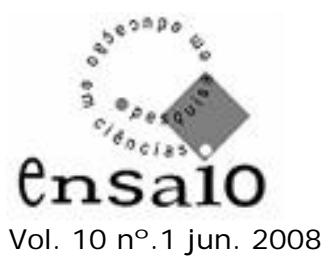

\section{Considerações iniciais}

Nos últimos anos, a sala de aula tem sido objeto de um grande número de pesquisas realizadas por diversas disciplinas. Na literatura especializada, são freqüentes estudos sobre assuntos como: análise do desempenho cognitivo dos alunos (SOARES et al., 2001); linguagem e formação de conceitos no ensino de ciências (MORTIMER, 2000); dinâmica discursiva presente em situações experimentais nos espaços escolares e não escolares (NASCIMENTO, 2002); dificuldades de ensino-aprendizagem na alfabetização (CASTANHEIRA e SANTIAGO, 2004); práticas discursivas argumentativas de alunos do laboratório didático de física (VILLANI, 2002); elaboração de material de apoio didático às práticas pedagógicas dos professores (CIRINO et al., 2004); análise de discurso na sala de aula de biologia (LIPARINI e MUNFORD, 2005), entre outras.

Dentre as várias possibilidades de abordagem da sala de aula, a análise do ambiente de aprendizagem tem sido uma das opções de trabalho no campo educacional. Nas últimas três décadas, pesquisas internacionais têm se dirigido para o desenvolvimento e uso de instrumentos para avaliar o ambiente de aprendizagem da sala de aula a partir da perspectiva do aluno. Os esforços de pesquisas envolvendo a avaliação e a investigação da percepção de aspectos do ambiente de aprendizagem da sala de aula estabeleceram esta área como um próspero campo de estudo (FRASER e WALBERG, 1991). Medidas da percepção são usadas hoje com freqüência, particularmente na investigação de amostras grandes de salas de aula (SHE e FISHER, 2000).

Essas pesquisas internacionais que utilizam questionários para avaliar o ambiente de aprendizagem da sala de aula envolvem questões como: exploração de maneiras nas quais os instrumentos de avaliação do ambiente de aprendizagem da sala de aula podem ser usados por psicólogos escolares (BURDEN e FRASER, 1993); investigação de mudanças no ambiente de aprendizagem da sala de aula durante a transição entre níveis de ensino diferentes (MIDGLEY et al., 1991); incorporação da avaliação do ambiente de aprendizagem da sala de aula nos esquemas de avaliação do professor (ELLETT, 1997);

pesquisas sobre o ambiente de aprendizagem do laboratório de ciências (FRASER et al., 1995); estudos transculturais do ambiente de aprendizagem da sala de aula em mais de um país (KHINE e FISHER, 2003); entre outras. 


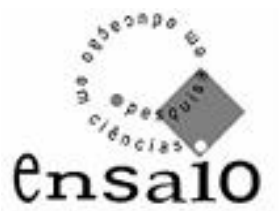

Vol. 10 no. 1 jun. 2008

Os estudos sobre o ambiente de aprendizagem apontam diversas vantagens no uso da percepção dos alunos como um indicador da qualidade do ambiente da sala de aula. Podem-se citar como exemplos (STODOLSKY, 1984; WALBERG e HAERTEL, 1980): os alunos estão diretamente envolvidos nas atividades da sala de aula e observam mais o comportamento típico do professor do que um observador externo (o comportamento do professor é dependente do contexto e pode variar); os alunos estão mais familiarizados com as idiossincrasias dos seus professores, que podem ser interpretadas diferentemente por um observador; os alunos estão em uma posição melhor para julgar certos aspectos do comportamento do professor, como, por exemplo, a clareza de expressão; o uso de observadores treinados durante um período de tempo é mais caro; a presença de observadores pode alterar o que geralmente acontece na sala de aula.

Nos últimos anos, o campo de estudo relacionado ao ambiente de aprendizagem da sala de aula tem passado por um notável crescimento, diversificação e internacionalização. Uma característica dessa área é a disponibilidade de uma variedade de questionários (válidos e econômicos quanto à aplicação) desenvolvidos especialmente para avaliar a percepção dos alunos do ambiente de aprendizagem da sala de aula (FRASER, 1998).

Segundo Dorman (2002), a maioria das pesquisas do ambiente de aprendizagem da sala de aula dos últimos anos tem sido conduzida nos Estados Unidos e na Austrália. Os instrumentos de avaliação do ambiente de aprendizagem da sala de aula originaram-se em países ocidentais, mas na última década os países asiáticos têm feito grandes contribuições. Alguns dos principais questionários desenvolvidos nos países ocidentais foram adaptados e validados para serem usados em vários países asiáticos (FRASER, 2002). Isso indica que esses países se tornaram grandes consumidores das pesquisas oriundas dos países ocidentais.

Nessa área, inúmeros trabalhos transculturais têm sido realizados em países como Holanda, Coréia, Indonésia, Índia, etc. Existem referências a pesquisas realizadas no continente africano, como, por exemplo, um estudo feito na África do Sul (SEBELA, 2003) e outro na Nigéria (IDIRIS e FRASER, 1994). 


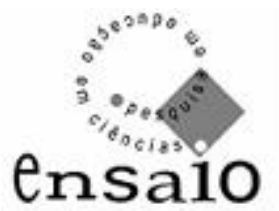

Vol. 10 no. 1 jun. 2008

Assim, a proposta desse artigo é analisar essa linha de pesquisa que trabalha com o desenvolvimento e uso de instrumentos para avaliar o ambiente de aprendizagem da sala de aula a partir da perspectiva do aluno.

Deve-se destacar que, por se tratar de uma linha de pesquisa muito específica, não foram encontrados estudos brasileiros no levantamento bibliográfico realizado. Existem estudos nacionais que relatam a utilização de instrumentos para avaliar diferentes aspectos do ambiente da sala de aula. Considerou-se, no entanto, que a inclusão desses estudos na linha de pesquisa abordada nesse artigo seria inadequada. Portanto, a maioria dos trabalhos aqui relatados é de origem estrangeira.

\section{A questão da terminologia}

Um aspecto recorrente na área de Ciências Humanas se faz presente nos estudos do tema abordado aqui: a questão da terminologia. Assim como outros termos da área de pesquisa social, o termo “ambiente de aprendizagem” carrega uma variedade de significados. Essa expressão tem sido usada para fazer referência ao ambiente psicossocial da sala de aula, ao gerenciamento da disciplina e das regras da sala de aula, à aquisição de habilidades sociais, etc. Além disso, o leitor que se interessar pela área poderá encontrar, por exemplo, outras expressões que se referem ao mesmo objeto de estudo: ambiente de aprendizagem da sala de aula, ambiente social da sala de aula, clima da sala de aula, clima social da sala de aula e ambiente da sala de aula. Deve-se destacar ainda que essa variedade de formas pode ser encontrada por vezes dentro de um mesmo texto.

Walker (2004) argumenta que a literatura especializada possui diferentes terminologias para se referir ao conceito de ambiente e indica que, no seu trabalho, esse conceito se refere exclusivamente ao ambiente psicossocial. O autor propõe a seguinte distinção: o termo ambiente psicossocial é utilizado quando faz referência ao ambiente de uma forma geral, e o termo ambiente de aprendizagem, quando se refere ao ambiente psicossocial na educação.

Nesse texto, será utilizada preferencialmente a expressão ambiente de aprendizagem da sala de aula, sempre para se referir ao ambiente psicossocial na educação; porém os 


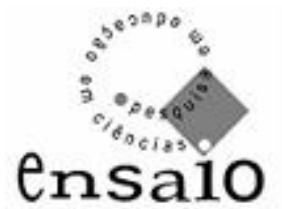

Vol. 10 no. 1 jun. 2008

outros termos citados aqui também podem aparecer no decorrer do texto. Quando isso acontecer, o sentido atribuído a eles será o mesmo explicitado acima.

\section{Uma consideração histórica}

Dorman (2002) e Walker (2004) remetem as raízes da pesquisa do ambiente de aprendizagem para 1920 e 1930, por meio da Teoria de Campo de Kurt Lewin, da teoria de personalidade de Henry Murray e consequentes desdobramentos desses trabalhos.

Neste texto, será abordado um período mais recente da pesquisa sobre o ambiente de aprendizagem: o final da década de 60 em diante. “A fase moderna da pesquisa do ambiente de aprendizagem começou no final da década de 1960 quando Rudolf Moos e Herbert Walberg começaram linhas independentes de pesquisa na conceitualização e avaliação de ambientes psicossociais” (DORMAN, 2002, p. 3).

Walker (2004) e Fraser (2002) apontam como os primeiros instrumentos usados para medir ambientes psicossociais na educação o Learning Environment Inventory (LEI), o Classroom Environment Scale (CES), o My Class Inventory (MCI) e o Class Activities Questionnaire (CAQ). Esses questionários são indicados como historicamente importantes e o início da elaboração deles data do final da década de 60. Ainda segundo os autores, os instrumentos contemporâneos de avaliação do ambiente de aprendizagem mais influentes são o Questionnaire on Teacher Interaction (QTI), o Science Laboratory Environment Inventory (SLEI), o Constructivist Learning Environment Survey (CLES) e o What is Happening in this Class ? (WIHIC) Questionnaire.

Além desses, diversos questionários podem ser citados (ANEXO A).

\section{Modalidades de pesquisa}

Pode-se considerar que os estudos envolvendo o ambiente de aprendizagem da sala de aula tendem a se enquadrar em modalidades diferentes. Com o objetivo de ilustrar as aplicações que os instrumentos de avaliação do ambiente de aprendizagem da sala de aula podem ter, serão consideradas algumas modalidades de pesquisa. Para Fraser (2002), as modalidades mais comuns são: associações entre os resultados dos alunos e o ambiente de aprendizagem da sala de aula; avaliação de inovações educacionais; diferenças entre as 


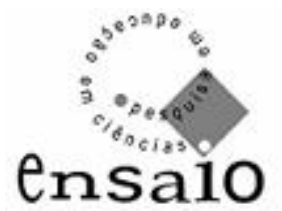

Vol. 10 no. 1 jun. 2008

percepções dos alunos e do professor de uma mesma sala de aula; determinantes do ambiente de aprendizagem da sala de aula; uso de métodos de pesquisa qualitativos; e estudos transculturais.

Isso não implica em dizer que não existam outras modalidades, embora sejam menos freqüentemente pesquisadas. Ainda vale lembrar que, em muitos estudos, aparecem duas ou mais modalidades ao mesmo tempo. Dessa forma, um pesquisador pode, por exemplo, realizar um estudo transcultural e ao mesmo tempo comparar as diferenças entre as percepções dos alunos e do professor de uma mesma sala de aula.

$\underline{\text { Associações entre os resultados dos alunos e o ambiente de aprendizagem da sala de aula }}$

Em relação ao início dessa modalidade de pesquisa sobre o ambiente da sala de aula, Walker (2004) afirma que Walberg e Moos começaram a considerar o ambiente psicossocial e sua influência nos resultados dos alunos no final da década de 60 e início da década de 70.

Considerando todas as modalidades citadas anteriormente, essa possui a tradição de pesquisa mais forte (FRASER, 2002). É muito comum encontrar na literatura da área associações entre variáveis do ambiente de aprendizagem e os resultados dos alunos, tanto no campo cognitivo quanto no afetivo. Pode-se considerar como resultados cognitivos as notas obtidas pelos alunos. Quando os resultados afetivos dos alunos, no entanto, são considerados, a questão se torna mais complexa. Definir o termo "afetivo" não é uma tarefa fácil porque esse pode suscitar uma variedade de significados. Nessa área de pesquisa, o termo aparece ligado basicamente ao estudo das atitudes dos alunos. Assim, pesquisas sobre o afeto dos alunos são relatadas como pesquisas atitudinais.

As atitudes são freqüentemente investigadas por meio da utilização de questionários. Pode-se citar, como exemplo, um instrumento que é usado em grande parte dos estudos da área: o Test of Science-Related Attitudes (TOSRA), elaborado por Barry J. Fraser (certamente um dos autores mais importantes da área). Esse teste às vezes é usado na sua totalidade e às vezes em partes, por meio da seleção de algumas escalas ou itens que mais interessam aos pesquisadores. Walker (2005), por exemplo, desenvolveu um instrumento chamado Test of Geography-Related Attitudes (ToGRA). O ToGRA se apropriou de três 


\section{ensaio}

Vol. 10 no. 1 jun. 2008

escalas do TOSRA fazendo modificações do ensino de ciências para o ensino de geografia.

O estudo das associações ambiente-resultado é feito normalmente por meio de métodos estatísticos. Algumas pesquisas envolvem o uso de mais de um questionário do ambiente de aprendizagem da sala de aula no mesmo estudo.

Portanto, os autores que trabalham nessa linha de pensamento empreendem esforços no sentido de não colocar uma ênfase muito grande no campo cognitivo e dar uma atenção maior também para resultados no campo afetivo. Além disso, procuram trazer evidências de que a percepção do ambiente de aprendizagem da sala de aula pode influenciar os resultados dos alunos. Alguns autores afirmam que essas associações já estão suficientemente comprovadas. Fraser (2002), por exemplo, cita uma tabulação de 40 estudos que mostrou esse tipo de associação fazendo uso de uma variedade de medidas de resultados cognitivos e afetivos. Foram utilizados nesses estudos diversos instrumentos de avaliação do ambiente de aprendizagem da sala de aula em vários países e níveis de ensino.

\section{Avaliação de inovações educacionais}

Uma outra possibilidade de pesquisa é a avaliação de inovações educacionais e novos currículos. Isso pode ser medido por meio do impacto causado pela implementação de programas educacionais e/ou novas diretrizes curriculares na transformação do ambiente de aprendizagem da sala de aula.

Burnley et al. (2002), por exemplo, desenvolveram nos Estados Unidos o questionário Statements About Science Instrument (SASI) para avaliar o impacto gerado por um programa de ciências da graduação que acontece no período de férias.

Kim et al. (1999) utilizaram o Constructivist Learning Environment Survey (CLES) para avaliar o impacto causado por um novo currículo geral de ciências na Coréia. O novo currículo foi baseado em uma visão construtivista. O currículo utilizado anteriormente também possuía uma ênfase nessa mesma teoria educacional. No entanto, algumas modificações foram feitas e novos livros escolares foram adotados. Devido a essas questões, o CLES foi o questionário escolhido. Os autores compararam salas de aula que 


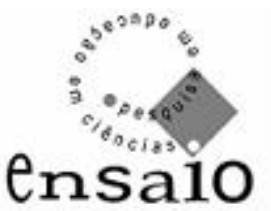

Vol. 10 no. 1 jun. 2008

foram expostas ao novo currículo com salas que não haviam sido expostas. As turmas nas quais o novo currículo foi trabalhado perceberam o ambiente de aprendizagem da sala de aula como "mais construtivista" do que aquelas turmas que não foram expostas à modificação curricular. O CLES possui cinco escalas que medem aspectos como: a participação dos alunos no planejamento, condução e avaliação do aprendizado; o envolvimento ativo dos alunos no processo investigativo, negociação em sala de aula ou discussão em grupo; a relevância do aprendizado para a vida dos alunos, etc. Considerase que escores mais altos nas escalas correspondem a uma percepção do ambiente de aprendizagem mais construtivista.

Considerou-se que a comparação entre essas salas de aula poderia trazer informações sobre o impacto do novo currículo geral de ciências da Coréia no caráter construtivista do ambiente de aprendizagem da sala de aula. Pode-se perceber que o método utilizado pelos pesquisadores para a avaliação foi uma comparação clássica do tipo grupo controle e grupo experimental.

Chen et al. (2002) utilizaram o What is Happening in this Class? (WIHIC) para avaliar uma reforma curricular em Taiwan. Nesse estudo, seis professores de ciências de escola pública desenvolveram um módulo interdisciplinar enquanto participavam de um programa de desenvolvimento profissional. Dois desses professores implementaram o módulo em cinco salas de uma escola. Os autores fizeram uma investigação da mudança na percepção dos alunos aplicando o questionário antes e depois da experiência do módulo. De forma geral, a percepção dos alunos do ambiente de aprendizagem da sala de aula se tornou mais positiva depois da implementação do módulo. Os alunos obtiveram escores mais altos nas diferentes escalas do questionário. Essa mudança foi atribuída à natureza diferente das tarefas de aprendizagem do módulo, às estratégias de ensino dos professores baseadas na teoria construtivista e à abordagem de ensino em equipe encorajada pela reforma curricular. Aqui, os autores utilizaram as mesmas turmas para estabelecer uma comparação, ao invés de usar turmas diferentes.

Deve-se destacar que, nos dois estudos citados nessa seção, os autores não explicitam qual foi a teoria de cunho construtivista utilizada. 


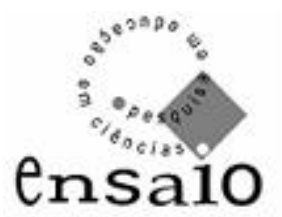

Vol. 10 no. 1 jun. 2008

\section{$\underline{\text { Diferenças entre as percepções dos alunos e do professor }}$}

Essa modalidade de pesquisa se focaliza nas diferenças entre as percepções dos alunos e do professor do ambiente real e do ambiente preferido de uma mesma sala de aula. O trecho a seguir explica o procedimento que é seguido nesses casos:

[...] essa linha de estudo envolve administrar um dado instrumento do ambiente de aprendizagem para medir o ambiente preferido dos alunos com uma versão preferida do instrumento. Isso é então seguido pela medida da percepção deles do ambiente real usando uma versão real do mesmo instrumento. Ao mesmo tempo, o professor responde a uma versão do professor do instrumento, assim produzindo três grupos de dados relacionados à percepção da mesma sala de aula. Cada versão do instrumento contém os mesmos itens, mas eles são formulados de uma maneira levemente diferente em cada versão ${ }^{1}$ (WALKER, 2004, p. 12, grifo do autor).

Geralmente, encontra-se o seguinte padrão de resultados: nesses estudos, os professores tendem a perceber o ambiente de aprendizagem da sala de aula de uma forma mais positiva do que os alunos; os alunos preferem um ambiente mais positivo àquele que realmente acontece na sala de aula.

Pode-se citar como exemplo um estudo feito por Quek et al. (2002) utilizando o Chemistry Laboratory Environment Inventory (CLEI). Os autores aplicaram esse instrumento em uma amostra de 497 alunos de três escolas de Cingapura e, por meio de duas versões do questionário (comparação entre o ambiente real e o ambiente preferido pelos alunos), chegaram, no resultado relatado acima, como mais freqüente: os alunos demonstraram preferir um ambiente mais positivo àquele que realmente acontece na sala de aula.

Deve-se destacar que nesse tipo de pesquisa freqüentemente existe a recomendação de uma aplicação prática: usar essas informações para a melhoria do ambiente de aprendizagem da sala de aula. Os professores podem comparar a visão deles com a dos alunos e promover modificações na sua aula. Assim, essas informações podem funcionar como uma fonte de reflexão para a prática pedagógica dos professores.

\footnotetext{
${ }^{1}$ Texto original em inglês.
} 


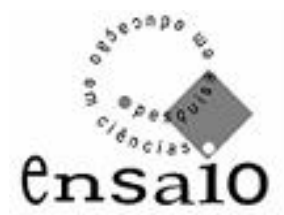

Vol. 10 no. 1 jun. 2008

$\underline{\text { Determinantes do ambiente de aprendizagem da sala de aula }}$

Dimensões do ambiente de aprendizagem da sala de aula têm sido usadas em pesquisas dirigidas para a identificação de como o ambiente de aprendizagem varia em função de fatores como a personalidade do professor, o gênero dos alunos, o gênero do professor, os níveis de ensino diferentes, o tamanho da sala, as disciplinas diferentes e o tipo de escola. Esses são exemplos de determinantes do ambiente de aprendizagem da sala de aula mais estudados. Na Ásia, por exemplo, o determinante do ambiente de aprendizagem da sala de aula mais pesquisado é sem dúvida o gênero dos alunos (FRASER, 2002). Geralmente, encontra-se o seguinte padrão de resultados nesses estudos que consideram o gênero dos alunos: as mulheres tendem a perceber o ambiente de aprendizagem da sala de aula de uma forma mais positiva do que os homens (KHINE e FISHER, 2003).

Pode-se citar como exemplo um estudo feito por She e Fisher (2002) utilizando o Teacher Communication Behavior Questionnaire (TCBQ). Os autores aplicaram esse instrumento em uma amostra de 1.049 alunos em Taiwan. Eles analisaram o gênero dos alunos e compararam as disciplinas de Física e Biologia, chegando aos seguintes resultados: as alunas perceberam o ambiente de aprendizagem da sala de aula de uma forma mais positiva do que os alunos; os professores de Biologia foram percebidos de uma maneira mais positiva do que os professores de Física. Considera-se que escores mais altos nas escalas do questionário correspondem a uma percepção do ambiente de aprendizagem mais positiva.

Uso de métodos de pesquisa qualitativos

A combinação de métodos quantitativos e qualitativos é uma prática freqüente nesse campo de trabalho. Geralmente, os pesquisadores usam, junto com o questionário sobre o ambiente de aprendizagem da sala de aula, métodos qualitativos, de uma maneira complementar. Entrevistas com alunos, professores, funcionários da escola ou familiares, gravação em vídeo das salas de aula, observação das aulas e utilização de diário de campo são alguns exemplos. Também é muito comum o uso de métodos qualitativos no pré-teste 


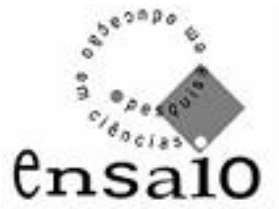

Vol. 10 no. 1 jun. 2008

do questionário que é feito com os alunos. São feitas entrevistas com pequenos grupos de estudantes para averiguar a adequação do questionário e o entendimento que eles têm do instrumento. Assim, modificações podem ser feitas antes do estudo envolver um trabalho em larga escala. Esse procedimento geralmente faz parte do desenvolvimento e validação dos questionários.

Pode-se citar como exemplo de combinação de métodos quantitativos e qualitativos um estudo feito por Waldrip e Fisher (2000), cujo artigo tem um título ilustrativo: The Development and Validation of a Learning Environment Questionnaire Using Both Quantitative and Qualitative Methods. Os autores avaliaram fatores culturais que afetam o ambiente de aprendizagem de alunos de ciências por meio do desenvolvimento e validação do Cultural Learning Environment Questionnaire (CLEQ). A amostra foi de 3.785 alunos da Austrália, totalizando 186 salas de 67 escolas. Esse trabalho visou o estudo do ambiente de aprendizagem de salas multiculturais. Entrevistas foram utilizadas para entender como os alunos interpretavam cada escala do questionário, o que eles pensavam sobre o significado das frases, as percepções divergentes dentro de uma mesma sala de aula, etc. Tudo isso como parte da validação do instrumento.

E, em relação à combinação desses métodos, Fraser (2002) destaca que: a) geralmente, os achados das entrevistas e observações replicam os achados do uso de questionários do ambiente de aprendizagem; b) as informações das entrevistas com alunos contribuem para esclarecer as suas repostas ao questionário; c) as entrevistas com os professores fornecem informações de background sobre a situação prática nas salas de aula e escolas.

Os estudos mostram, portanto, uma congruência entre os resultados dos métodos quantitativos e qualitativos. Além disso, a utilização de recursos, como as entrevistas, fornecem informações valiosas sobre o contexto da escola, as respostas dos alunos, etc.

\section{Estudos transculturais}

Estudos transculturais utilizando instrumentos de avaliação do ambiente de aprendizagem da sala de aula com amostras de diferentes países têm sido amplamente conduzidos . Essa questão é de grande importância nessa área de pesquisa. 


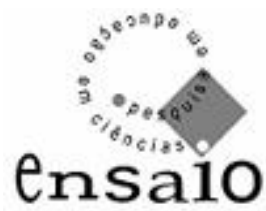

Vol. 10 no. 1 jun. 2008

Nos últimos anos, pesquisadores desenvolveram vários questionários para avaliar a percepção dos alunos do seu ambiente de aprendizagem da sala de aula. Esses questionários têm sido usados em diferentes países e em níveis diferentes de ensino por centenas de pesquisadores, milhares de professores e milhões de alunos pelo mundo (FRASER, 2002).

A colaboração entre pesquisadores tem sido cada vez maior. Khine e Fisher (2003), por exemplo, fizeram associações entre a percepção dos alunos das interações professoraluno e medidas atitudinais relativas à ciência. Eles aplicaram o Questionnaire on Teacher Interaction (QTI) em uma amostra de 1.188 alunos, sendo 543 homens e 645 mulheres de 54 salas de dez escolas. Um dos objetivos desse estudo foi conseguir dados para a validação do QTI em Brunei. O primeiro autor é de uma Universidade de Cingapura e o segundo de uma Universidade da Austrália.

Aldridge et al. (1999) fizeram um estudo conjunto na Austrália e em Taiwan com o objetivo de observar as diferenças e similaridades entre a percepção dos alunos de cada país. Esse trabalho envolveu seis pesquisadores australianos e sete de Taiwan. O questionário utilizado, cujo processo de desenvolvimento e validação aconteceu nos dois países, foi o What is Happening in this Class? (WIHIC). Esse instrumento de avaliação do ambiente de aprendizagem foi utilizado como um ponto de partida para a coleta de dados qualitativos, como observações de sala de aula e entrevistas com professores e alunos. A amostra na Austrália foi de 1.081 alunos em 50 salas de 25 escolas. Em Taiwan, o número de alunos foi de 1.879 em 50 salas de 25 escolas.

Deve-se destacar que os pesquisadores que trabalham com estudos transculturais usando instrumentos de avaliação do ambiente de aprendizagem da sala de aula demonstram estar atentos para as questões relacionadas às diferenças culturais. Aldridge et al. (1999), por exemplo, destacam a necessidade de cautela na interpretação de resultados do questionário de dois países com diferenças culturais. Fraser (2002) cita um estudo feito na Austrália e Cingapura: os professores australianos foram percebidos como dando mais responsabilidade e liberdade para os alunos em comparação com os professores de Cingapura, que foram vistos como mais rigorosos. Segundo o autor, esses resultados não são uma surpresa por causa das diferenças entre o background cultural e o 
sistema educacional dos dois países. Já Walker (2004) demonstra que a percepção dos alunos do seu ambiente de aprendizagem é influenciada por fatores sócio-culturais e recomenda cuidado no uso de instrumentos originalmente desenvolvidos no Ocidente em investigações transculturais.

\section{O presente e o futuro das pesquisas sobre o ambiente de aprendizagem da sala de aula}

Em relação às pesquisas atuais sobre o ambiente de aprendizagem, uma revisão de artigos apresentados no encontro de 2002 da American Educational Research Association (AERA) indica três temas gerais para a pesquisa contemporânea do ambiente de aprendizagem da sala de aula: ambientes de aprendizagem multimídia, desenvolvimento de instrumentos, e introdução do estudo do ambiente de aprendizagem em outros países (DORMAN, 2002).

Quanto aos trabalhos sobre ambientes de aprendizagem multimídia, alguns questionários como o Constructivist Multimedia Learning Environment Survey (CMLES) são exemplos de pesquisas contemporâneas. Atualmente, vários instrumentos estão direcionados para ambientes tecnológicos. Já o desenvolvimento de instrumentos possui grande força. Isso ficou evidenciado por meio dos exemplos desse texto.

Sobre as tendências dos trabalhos sobre o ambiente de aprendizagem, Dorman (2002) aponta quatro possíveis direções futuras para a pesquisa nesse campo: 1) uma necessidade de fornecer avaliações mais abrangentes dos ambientes de aprendizagem das salas de aula e das escolas; 2) a articulação do ambiente da sala de aula com outros ambientes de aprendizagem; 3) a avaliação do ambiente de aprendizagem da sala de aula deve ter um papel em projetos amplos e abrangentes onde o ambiente não seja o único foco da investigação; 4) uma recomendação da utilização de métodos mais robustos de análise de dados, como, por exemplo, o uso da análise fatorial confirmatória.

A primeira direção está relacionada à realidade de países desenvolvidos. Trata-se, nesse caso, da inserção cada vez maior de tecnologia na sala de aula. Existem, por exemplo, estudos sobre a utilização de laptops nas aulas de ciências. Para aulas desse tipo, as condições do ambiente físico têm se mostrado importantes, principalmente a 


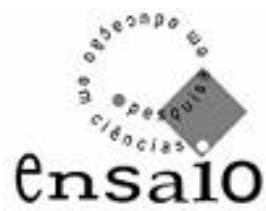

Vol. 10 no. 1 jun. 2008

questão dos materiais disponíveis. A segunda direção trata da articulação do ambiente da sala de aula com outros ambientes de aprendizagem, como, por exemplo, a família e grupos fora da escola. A terceira direção enfatiza que o ambiente de aprendizagem da sala de aula precisa ser incluído em projetos de pesquisa mais amplos, sendo um elemento a mais na pesquisa, e não o único elemento. A última direção chama a atenção para o pouco uso da análise fatorial confirmatória nos estudos sobre ambiente de aprendizagem.

Deve-se destacar também que as pesquisas transculturais vêm demonstrando resultados importantes e promissores e, por isso, continuarão a fazer parte da agenda futura dessa área.

Além disso, como uma outra tendência para trabalhos posteriores, os pesquisadores também estão buscando uma interlocução com outras disciplinas, para produzir uma pesquisa realmente transdisciplinar que influencie os estudos sobre o ambiente de aprendizagem (WALKER, 2004). Um exemplo dessa questão é o desenvolvimento do Cultural Learning Environment Questionnaire (CLEQ) (WALDRIP e FISHER, 2000). Nesse questionário, aspectos tradicionais do ambiente de aprendizagem foram integrados a aspectos culturais extraídos de disciplinas como a Antropologia e a Sociologia.

Assim, essa revisão da literatura demonstra a riqueza de um campo de pesquisa que continua em evolução: diversas possibilidades de trabalho, grupos de pesquisadores de vários países em um esforço conjunto e muitas aplicações práticas para todos os níveis educacionais. Foram encontradas publicações da área nos mais diversos periódicos de educação. Também existe um periódico dedicado exclusivamente a trabalhos sobre o ambiente de aprendizagem chamado Learning Environments Research (LER).

Pode-se dizer que os pesquisadores da área traçaram uma base forte para trabalhos futuros. Isso foi obtido por meio do desenvolvimento, tradução e validação de uma variedade enorme de instrumentos de avaliação do ambiente de aprendizagem da sala de aula. Dessa forma, pesquisadores e professores de diversas nacionalidades e línguas podem fazer uso desses questionários de acordo com seus objetivos. 


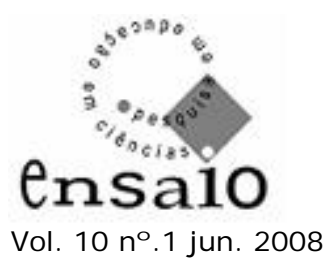

\section{Referências}

Vol. 10 no. 1 jun. 2008

ALDRIDGE, J. M.; FRASER, B. J.; HUANG, T.C.I. Investigating classroom environments in Taiwan and Australia with multiple research methods. Journal of Educational Research, 93, p. 48-62, 1999.

BURDEN, R.; FRASER, B. J. Use of classroom environment assessments in school psychology: A British perspective. Psychology in the Schools, 30, p. 232-240, 1993.

BURNLEY, P. C.; EVANS, W.; JARRETT, O. S. A comparison of approaches and instruments for evaluating a geological sciences research experiences program. Journal of Geoscience Education, v. 50, n. 1, p. 15-24, January. 2002.

CASTANHEIRA, M. L.; SANTIAGO, A. L. Oralidade e escrita: dificuldades de ensinoaprendizagem na alfabetização. Boletim Salto Para o Futuro, Rio de Janeiro, p. 14-22, 2004.

CHEN, H.; CHANG, W.; CHANG, H. Different Gender Students' Perceptions of Classroom Climate in a Trial of a Teacher Developed Interdisciplinary Module. Proc. Natl. Sci. Counc., v. 12, n. 3, p. 79-90, 2002.

CIRINO, S. D.; EITERER, C. L.; GUIMARÃES, E. J. Laboratório de Produção de Material Didático - PROMAD. In: XII ENCONTRO NACIONAL DE DIDÁTICA E PRÁTICA DE ENSINO - ENDIPE, 2004, Curitiba. Anais do XII Encontro Nacional de Didática e Prática de Ensino - ENDIPE, 2004. v.1. p. 7887-7896.

DORMAN, J. Classroom environment research: Progress and possibilities. Queensland Journal of Educational Research, 18(2), p. 112-140, 2002. Disponível em: <http:// education.curtin.edu.au/iier/qjer/qjer18/dorman.html>. Acesso em: 7 set. 2005.

ELLETT, C. D. Classroom-based assessments of teaching and learning. In: STRONGE, J. Evaluating teaching: A guide to current thinking and best practice. Newbury Park, CA: Corwin, 1997. p. 107-128.

FRASER, B. J. Classroom environment instruments: Development, validity and applications. Learning Environments Research, 1, p. 7-33, 1998.

FRASER, B. J. Learning environments research: yesterday, today and tomorrow. In: GOH, S. C.; KHINE, M. S. (Eds.) Studies in educational learning environments: an international perspective. River Edge, NJ: World Scientific, 2002. p. 1-25.

FRASER, B. J.; GIDDINGS, G. J.; MCROBBIE, C. J. Evolution and validation of a personal form of an instrument for assessing science laboratory classroom environments. Journal of Research in Science Teaching, 32, p. 399-422, 1995.

FRASER, B. J.; WALBERG, H. J. Educational environments: Evaluation, antecedents and consequences. Oxford, England: Pergamon Press, 1991.

IDIRIS, S.; FRASER, B. J. A Study of Learning Environments in Agricultural Science Classrooms in Nigeria. In: ANNUAL CONFERENCE OF THE AUSTRALIAN

ASSOCIATION FOR RESEARCH IN EDUCATION (AARE), 1994, The University of Newcastle, New South Wales. Anais da Annual conference of the Australian Association for Research in Education (AARE), 1994.

KHINE, M. S.; FISHER, D. L. Teacher-Student Interactions in Science Classrooms in Brunei. Journal of Classroom Interaction, v. 38, n. 2, p. 21-28, 2003. 


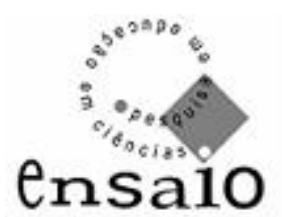

Vol. 10 no. 1 jun. 2008

KIM, H. B.; FISHER, D. L.; FRASER, B. J. Assessment and investigation of Constructivist Science Learning Environments in Korea. Research in Science \& Technological Education, v. 17, n. 2, p. 239-249, 1999.

LIPARINI, A.; MUNFORD, D. Análise de Discurso Dentro da Sala de Aula e a Influência da Metodologia Tempestade de Idéias no Processo de Aprendizagem dos Alunos. In: I ENCONTRO NACIONAL DE ENSINO DE BIOLOGIA, 2005, Rio de Janeiro. Anais do I Encontro Nacional de Ensino de Biologia, 2005. p. 602-606.

MIDGLEY, C.; ECCLES, J. S.; FELDLAUFER, H. Classroom environment and the transition to junior high school. In: FRASER, B. J.; WALBERG, H. J. Educational environments: Evaluation, antecedents and consequences. Oxford, England: Pergamon Press, 1991. p. 113-140.

MORTIMER, E. F. Linguagem e formação de conceitos no ensino de ciências. Belo Horizonte: Ed. UFMG, 2000. 383 p.

NASCIMENTO, S. S. A dinâmica discursiva em situações experimentais em espaços escolares e não escolares. Relatório de pesquisa. Pro Reitoria de Pesquisa. UFMG, 2002. QUEK, C. L.; WONG, A. F. L.; FRASER, B. J. Gender differences in the perceptions of chemistry laboratory classroom environments. Queensland Journal of Educational Research, 18(2), p. 164-182, 2002. Disponível em: <http://education.curtin.edu.au/ iier/qjer/qjer18/quek.html>. Acesso em: 15 nov. 2005.

SEBELA, M. P. Using teacher action research to promote constructivist learning environments in mathematics classes in South Africa. 2003. Thesis (Doctor of Mathematics Education) - Science \& Mathematics Education Centre, Curtin University of Technology, 2003.

SHE, H. C.; FISHER, D. The development of a questionnaire to describe science teacher communication behavior in Taiwan and Australia. Science Education, 84, p. 706-726, 2000.

SHE, H. C.; FISHER, D. Teacher Communication Behavior and its Association With Students' Cognitive and Attitudinal Outcomes in Science in Taiwan. Journal of Research in Science Teaching, v. 39, n. 1, p. 63-78, 2002.

SOARES, J. F.; ALVES, M. T. G.; OLIVEIRA, R. M. O efeito de 248 escolas de nível médio no vestibular da UFMG nos anos de 1998, 1999 e 2000. Estudos em Avaliação Educacional, n. 24, p. 69-117, Jul-Dez. 2001.

STODOLSKY, S. Teacher evaluation: The limits of looking. Educational Researcher, 13, p. 11-18, 1984.

VILLANI, C. E. P. As práticas discursivas argumentativas de alunos do ensino médio no laboratório didático de física. 2002. Dissertação (Mestrado em Educação) - Faculdade de Educação, Universidade Federal de Minas Gerais, Belo Horizonte, 2002.

WALBERG, H. J.; HAERTEL, G. D. Validity and use of educational environment assessments. Studies in Educational Evaluation, 6, p. 225-238, 1980.

WALDRIP, B. G.; FISHER, D. L. The Development and Validation of a Learning Environment Questionnaire Using Both Quantitative and Qualitative Methods. Journal of Classroom Interaction, v. 35, n. 2, p. 25-37, 2000.

WALKER, S. L. Learning environment research: A review of the literature (Learning Environments Monograph No. 2). San Marcos, TX: Texas State University - San Marcos, 


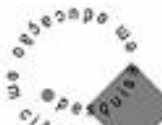 \\ ensa10}

Vol. 10 o.. 1 jun. 2008

Geography Department, 2004. Disponível em: <http:/uweb.txstate.edu/ sw36/ monographs>. Acesso em: 14 ago. 2005.

WALKER, S. L. The Test of Geography-Related Attitudes (ToGRA): A New Research Instrument. In: FOURTH INTERNATIONAL CONFERENCE ON SCIENCE, MATHEMATICS, AND TECHNOLOGY EDUCATION, 2005, Victoria, British Columbia. Anais da Fourth International Conference on Science, Mathematics, and Technology Education, 2005. p. 25-28.

DATA DE RECEBIMENTO: 24/03/2007

DATA DE APROVAÇÃO: 06/09/2007

DATA DE VERSÃO FINAL: 20/09/2007 


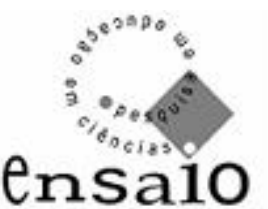

Vol. 10 no. 1 jun. 2008

\section{ANEXO A - Instrumentos de avaliação do ambiente de aprendizagem da sala de}

\section{aula}

São outros exemplos de instrumentos de avaliação do ambiente de aprendizagem da sala de aula o Cultural Learning Environment Questionnaire (CLEQ), o Student Perception Inventory (SPI), o Teacher Communication Behavior Questionnaire (TCBQ), o College and University Classroom Environment Inventory (CUCEI), o Individualized Classroom Environment Questionnaire (ICEQ), o New Classroom Environment Instrument (NCEI), o Chemistry Laboratory Environment Inventory (CLEI), o Chemistry Attitudes and Experiences Questionnaire (CAEQ), o Geography Classroom Environment Inventory (GCEI), o Test of Geography-Related Attitudes (ToGRA), o Computer Laboratory Environment Instrument (CLEI), o Computer-Facilitated Learning (CFL), o Constructivist Multimedia Learning Environment Survey (CMLES), o Technology-Rich Outcomes-Focused Learning Environment Inventory (TROFLEI), o Computerized Classroom Ergonomic Inventory (CCEI), o Metacognitive Orientation Learning Environment Scale - Science (MOLES-S), entre outros. 